\title{
Patient Portals: Objectives, Acceptance, and Effects on Health Outcome - A Scoping Review of Reviews
}

\author{
I. LEB $^{\mathrm{a}}$, S. MAGNIN ${ }^{\mathrm{b} 1}$ H.-U. PROKOSCH ${ }^{\mathrm{a}}$ and M. BOEKER ${ }^{\mathrm{c}}$ \\ ${ }^{a}$ Friedrich-Alexander-University Erlangen-Nürnberg, Chair of Medical Informatics \\ b Medical Data Integration Center, Dept. for IT and Applied Medical Informatics, Uni- \\ versity Hospital Tübingen, Translational Bioinformatics, University of Tübingen \\ Institute for Bioinformatics and Medical Informatics, University of Tübingen \\ ${ }^{\mathrm{c}}$ Technical University of Munich, School of Medicine, Medical Center rechts der Isar, \\ Institute for Medical Informatics, Statistics and Epidemiology-IMedIS
}

\begin{abstract}
Patient portals provide patients access to their electronic health record and other functions as secure messaging. For over a decade, more and more patient portals are developed for various settings. The aim of this scoping review of reviews is to systematically search the literature for existing reviews to provide an overview of patient portals' objectives, acceptance and effects on outcome. We followed the PRISMA Statement and its extension for scoping reviews, and searched for articles published in $2011-2021$. The 19 included articles were considerably heterogeneous concentrating on health outcome or patient portal facilitators and barriers.
\end{abstract}

Keywords. patient portal, electronic health record, personal health record, systematic review

\section{Introduction}

With the effect of the "Krankenhauszukunftsgesetz" (KHZG) hospitals shall receive a digital update including sponsoring for emergency capacities and digital infrastructure. One of these elements are patient portals[1]. Patient portals provide several functions for patients to access and/or manage health information via a secure online website with 24hour access such as recent visits, discharge summaries, medications. In addition, patient portals can enable the patient to securely communicate with physicians, request prescription refills, schedule appointments etc.[2]. By providing the opportunity to empower the patient to take over an active role in his/her own care [3], several studies came to the conclusion that patient portals and the patient engagement within can improve health outcomes or medication adherence[4]. However, the adoption rates are very low [5].

Many reviews already focused on various topics concerning patient portals. The aim of this scoping review of reviews is to search the literature for those existing reviews to provide an overview of patient portals' objectives, acceptance and effects on outcome.

${ }^{1}$ Ines Leb, Friedrich-Alexander-Universität Erlangen-Nürnberg, Wetterkreuz 15, 91058 Erlangen, EMail: ines.leb@fau.de 


\section{Methods}

The protocol follows the PRISMA Statement [6] and its extension for scoping reviews [7]. It has not been published, but can be supplied by the authors. Studies fulfilling all the following eligibility criteria were included:

1. The patient portal is connected to a hospital information system.

2. The study investigates and describes at least one of the following characteristics of patient portals: structure, development, use or influence on the health status.

3. The type of study is systematic review or meta-analysis according to the PRISMA-Statement and the Cochrane Handbook of Systematic Reviews.

4. The study reports on data items listed below.

The Web of Science (Clarivate Analytics) was searched in the configuration "All databases" on March $15^{\text {th }} 2021$. The search strategy is shown in Fig. 1.

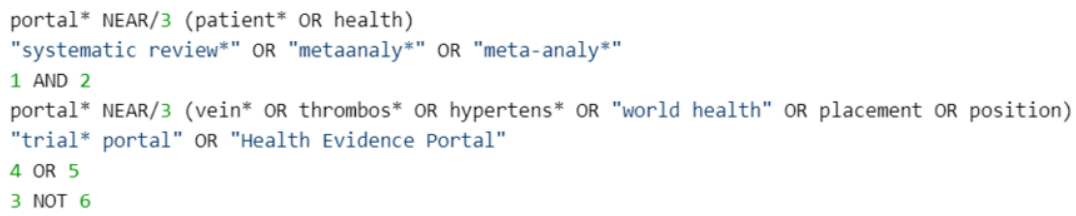

Figure 1: Search Query

The search was restricted to articles published between 2011 and 2021. For study inclusion the following steps were performed: (1) Titles and abstracts were scanned for mentioning of "portal" in the meaning of an internet portal and English language; (2) abstracts were scanned for the mentioning of a connection between portal and central hospital information system; (3) remaining full texts were checked for full concordance with the eligibility criteria described above. All steps were performed at least by two authors and ambiguous decisions were discussed by all authors until consensus. The following data items were extracted from the included reviews (Tab. 1):

Table 1: Data Extraction Items

\begin{tabular}{|l|l|l|}
\hline Item & Value & Explanation \\
\hline $\begin{array}{l}\text { Literature } \\
\text { Search }\end{array}$ & Time period & $\begin{array}{l}\text { For which time period was the liter- } \\
\text { ature search performed? }\end{array}$ \\
\hline Studies included & Number of studies & $\begin{array}{l}\text { How many studies were included in } \\
\text { the review? }\end{array}$ \\
\hline Health problem & Type of disease & $\begin{array}{l}\text { Is a specified kind of health problem } \\
\text { addressed? }\end{array}$ \\
\hline Targeted Effect & $\begin{array}{l}\text { Objective: e.g. improvement of patient-phy- } \\
\text { sician interaction }\end{array}$ & $\begin{array}{l}\text { What is the health-related objec- } \\
\text { tive/targeted effect of the portal? }\end{array}$ \\
\hline $\begin{array}{l}\text { Type of study } \\
\text { outcome }\end{array}$ & $\begin{array}{l}\text { Type of outcome: e.g. usability, acceptance, } \\
\text { health outcome, patient reported outcome }\end{array}$ & $\begin{array}{l}\text { What has been measured/investi- } \\
\text { gated in the study? }\end{array}$ \\
\hline Functions & $\begin{array}{l}\text { Type of functionalities: e.g. secure messag- } \\
\text { ing }\end{array}$ & $\begin{array}{l}\text { Which functions are available in the } \\
\text { portal? }\end{array}$ \\
\hline
\end{tabular}


Categories for extracted values were defined by all authors so that each value is covered by one category and articles can be described most expressive with them. Articles were categorized and grouped according to the reported data items as shown in Tab. 1.

\section{Results}

From the initial search result of 89 articles, 19 articles were included for data extraction. The PRISMA diagram (Fig. 2) shows detailed reasons for exclusion.

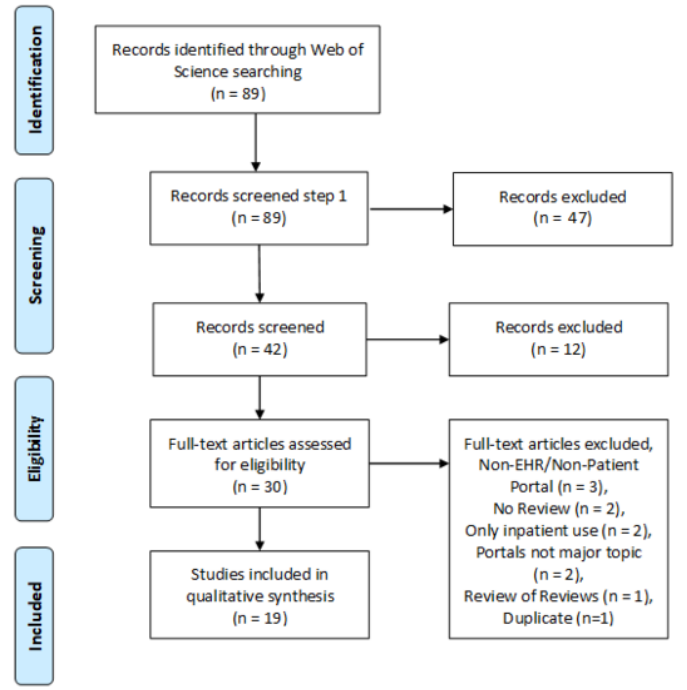

Figure 2: PRISMA diagram

Since most of the reviewed articles did not specify the health problem addressed and only mentioned chronic illnesses in general or vulnerable population [4,5,7-15], we did not include this column in the summary table (Fig 3a-c). However, four articles addressed diabetes [16-19].

The following targeted objectives were found: 9 articles described effects on health outcome $[8,11-13,16,19-22], 8$ the usage of patient portals $[4,5,11-13,15,17,20], 8$ facilitators $[4,5,9,11,13,14,17,21]$, and 7 barriers [4,5,9,11,14,17,21]. Additionally, characteristics of the users $[9,11,15,22]$, their adherence [11,12], patient empowerment [11], and effects on decision making [11]. Three reviews focused on portal design [4,10,15] and one on Meaningful Use [15].

The associated review outcomes can be grouped by three major topics: health outcome, facilitators and barriers: For health outcome 7 reviews reported an improved outcome $[8,12,16,18-20,22]$ and 4 better adherence [12,16,22,23], however 3 reported insufficient evidence or no improvement $[11,19,22]$. 4 reviews declared better patient satisfaction and empowerment $[11,12,18,23]$.

Facilitators mainly concentrate on assistance, training and provider/family engagement $[9,10,13,18,21,23]$ as well as good and user-centered design of the portal $[4,10,13,14,21]$, sociodemographic factors, e.g. younger users $[4,5,9,11,17,21]$, reminding users $[10,14]$ and adequate policy strategies $[10,14,21]$. 
Most concerning barriers reported in the reviews were privacy and security concerns $[4,5,9,10,13,23]$, limited access to the internet $[5,13,14,17]$, lack of technology experience [17,21], literacy [14], lack of interest [5,9], and awareness [21,23].

Functions of reviewed patient portals contained secure messaging [4,9$14,16,17,19,20,22-24]$, access to EHR-data (e.g. lab results) [9-14,16,17,19,23], medication refills [11,12,14,16,17,22], appointment booking [10-13,16,17], patient education $[11,12,14,16,17,22]$, add information $[4,9,13,14]$, decision support $[14,22,23]$. For detailed results see Figure 3a, b, c.

\section{Discussion}

This scoping review examined 19 systematic reviews with different themes and variations in the setting and outcome. Most reviews concentrated on health outcome or patient portal facilitators and barriers. Nevertheless, we found an overlap in facilitators such as patient training and barriers such as privacy concerns.

Ammenwerth et al concluded in 2012 that the impact of patient portals is only limited [12]. Consistently, Goldzweig et al added that no sufficient evidence on health outcome, cost, or utilization can be found. However, some studies stated that patient portals can be beneficial for healthcare [4]. Especially in the field of diabetes clinical parameters and so the health outcome could be improved [16,18,22], although those reviews also reported insufficient evidence.

To improve patient portal acceptance and usage sociodemographic factors have to be overcome [11], training and pc/internet access has to be provided [5,9,10,21]. Frequent reminder and provider encouragement also have an impact on the regularly use of patient portals [14].

Although the reviews concentrated on various health problems, the patient portals had similar functions, which validates the findings of Goldzweig et al.

Dendere et al suggest to develop standardized outcome assessment and studies focusing on objective outcomes for a comprehensive evaluation of patient portals [4]. This review is limited to patient portals which are directly connected to hospital EHR systems. Although this scoping review is methodically limited, we followed the PRISMA statement for systematic reviews as closely as possible. A quality assessment of the included reviews will be included in future work.

\section{Conclusion}

With this scoping review of reviews, we provide an overview of the current research on patient portals and the opportunities they present for patient care. In addition, our review shows that more research is needed on the use and use cases of patient portals in order to gain insights into problems that arise and their solutions, such as increased usability.

\section{Declarations and Acknowledgment}

Conflict of Interest: The authors declare that there is no conflict of interest. This work was funded by the German Federal Ministry of Science (BMBF) in the funding scheme digital progress hubs (01ZZ2102A/B) 


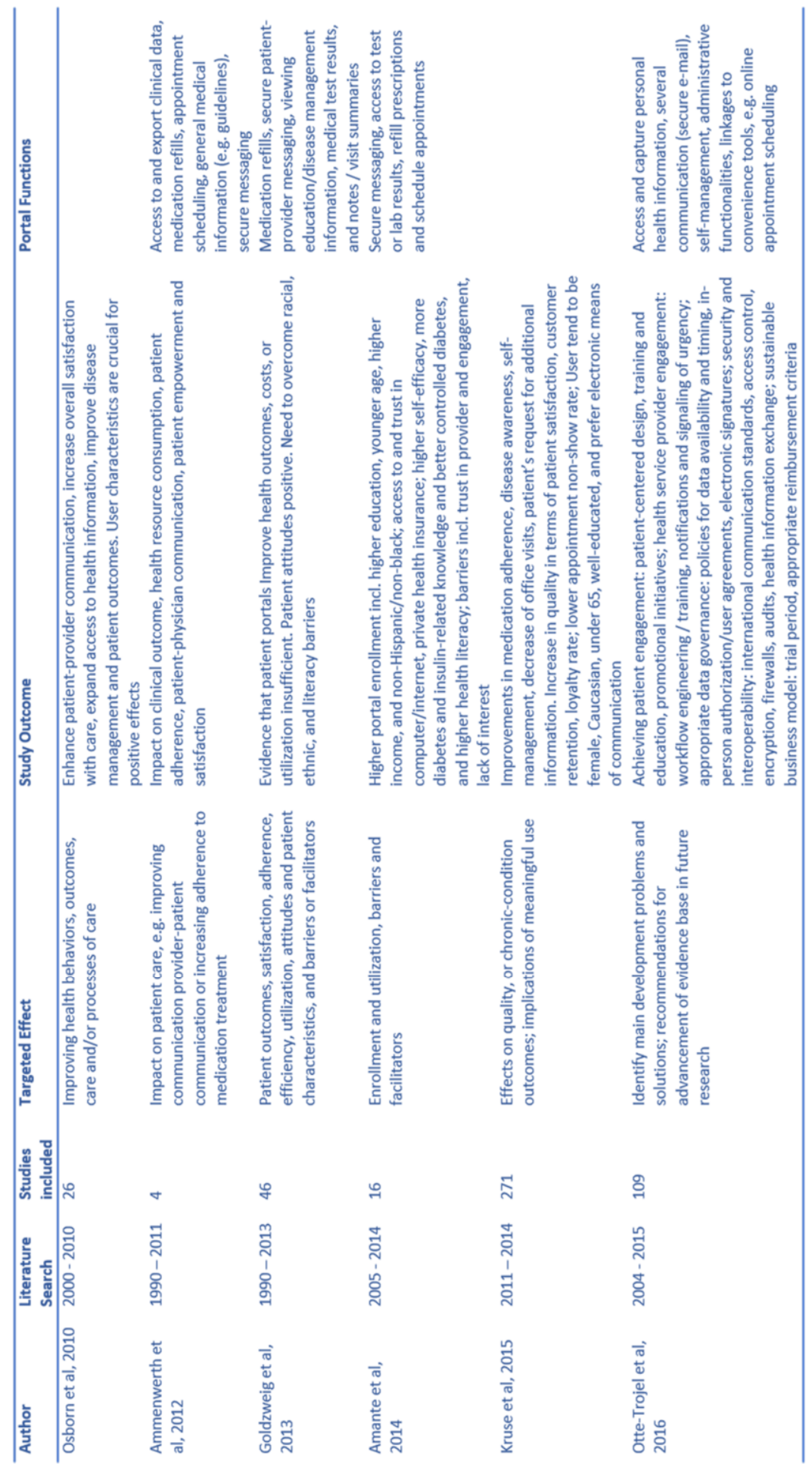

Figure 3a: Summary of findings in the reviewed literature 


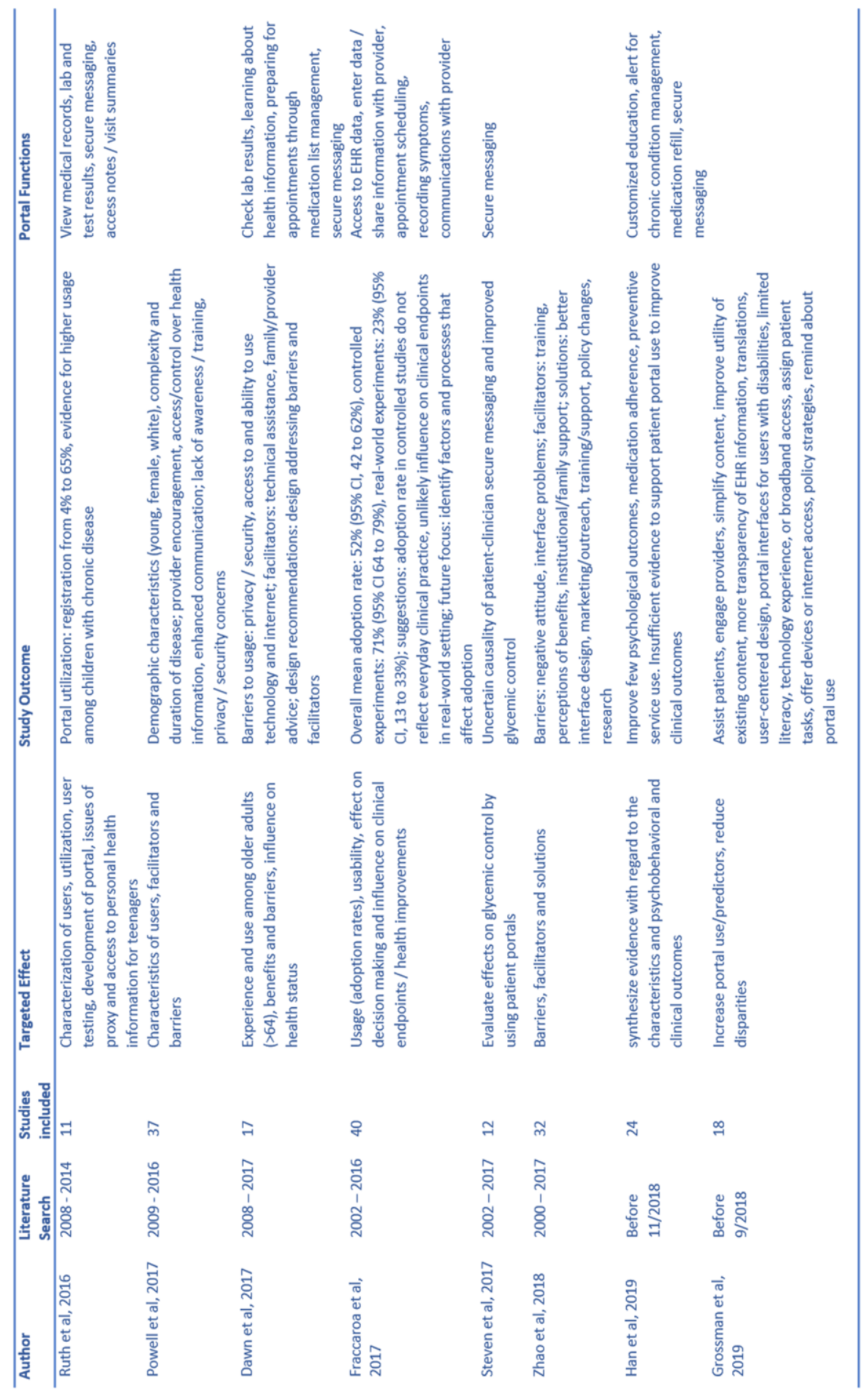

Figure 3b: Summary of findings in the reviewed literature 


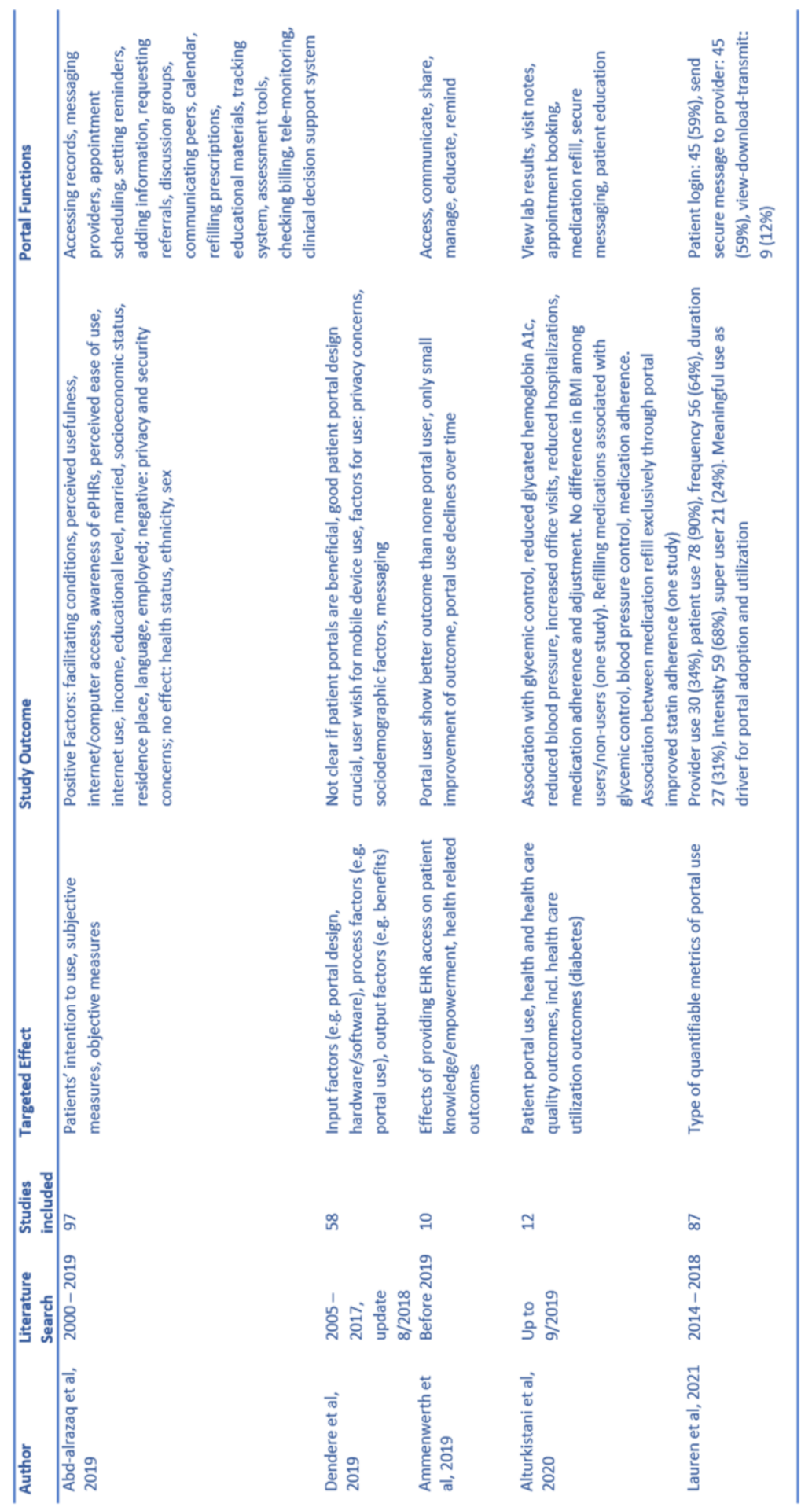

Figure 3c: Summary of findings in the reviewed literature 


\section{References}

[1] Bundesgesundheitsministerium, Krankenhauszukunftsgesetz (KHZG), https://www.bundesgesundheitsministerium.de/krankenhauszukunftsgesetz.html [cited 2021 March 31].

[2] What is a patient portal? | HealthIT.gov, https:/www.healthit.gov/faq/what-patient-portal [cited 2021 March 31].

[3] E. Ammenwerth, P. Schnell-Inderst, and A. Hoerbst, Patient Empowerment by Electronic Health Records: First Results of a Systematic Review on the Benefit of Patient Portals. Stud Health Technol Inform 165 (2011), 63-67.

[4] R. Dendere, C. Slade, and A. Burton-Jones, et al., Patient Portals Facilitating Engagement With Inpatient Electronic Medical Records: A Systematic Review. J Med Internet Res 21 (2019), e12779.

[5] A.A. Abd-Alrazaq, B.M. Bewick, and T. Farragher, et al., Factors that affect the use of electronic personal health records among patients: A systematic review. Int J Med Inform 126 (2019), 164175.

[6] D. Moher, A. Liberati, and J. Tetzlaff, et al., Preferred reporting items for systematic reviews and meta-analyses: the PRISMA statement. PLoS Med 6 (2009), e1000097.

[7] A.C. Tricco, E. Lillie, and W. Zarin, et al., PRISMA Extension for Scoping Reviews (PRISMAScR): Checklist and Explanation. Ann Intern Med 169 (2018), 467-473.

[8] E. Ammenwerth, A. Hoerbst, and S. Lannig, et al., Effects of Adult Patient Portals on Patient Empowerment and Health-Related Outcomes: A Systematic Review. Stud Health Technol Inform 264 (2019), 1106-1110.

[9] K.R. Powell, Patient-Perceived Facilitators of and Barriers to Electronic Portal Use: A Systematic Review. Comput Inform Nurs 35 (2017), 565-573.

[10] T. Otte-Trojel, A. de Bont, and T.G. Rundall, et al., What do we know about developing patient portals? a systematic literature review. J Am Med Inform Assoc 23 (2016), e162-8.

[11] C.L. Goldzweig, G. Orshansky, and N.M. Paige, et al., Electronic patient portals: evidence on health outcomes, satisfaction, efficiency, and attitudes: a systematic review. Ann Intern Med 159 (2013), $677-687$.

[12] E. Ammenwerth, P. Schnell-Inderst, and A. Hoerbst, The impact of electronic patient portals on patient care: a systematic review of controlled trials. J Med Internet Res 14 (2012), e162.

[13] D.K. Sakaguchi-Tang, A.L. Bosold, and Y.K. Choi, et al., Patient Portal Use and Experience Among Older Adults: Systematic Review. JMIR Med Inform 5 (2017), e38.

[14] L.V. Grossman, R.M. Masterson Creber, and N.C. Benda, et al., Interventions to increase patient portal use in vulnerable populations: a systematic review. J Am Med Inform Assoc 26 (2019), 855870 .

[15] R.A. Bush, C.D. Connelly, and M. Fuller, et al., Implementation of the Integrated Electronic Patient Portal in the Pediatric Population: A Systematic Review. Telemed J E Health 22 (2016), 144-152.

[16] A. Alturkistani, A. Qavi, and P.E. Anyanwu, et al., Patient Portal Functionalities and Patient Outcomes Among Patients With Diabetes: Systematic Review. J Med Internet Res 22 (2020), e18976.

[17] D.J. Amante, T.P. Hogan, and S.L. Pagoto, et al., A systematic review of electronic portal usage among patients with diabetes. Diabetes Technol Ther 16 (2014), 784-793.

[18] C.Y. Osborn, L.S. Mayberry, and S.A. Mulvaney, et al., Patient web portals to improve diabetes outcomes: a systematic review. Curr Diab Rep 10 (2010), 422-435.

[19] S.S. Coughlin, L.B. Williams, and C. Hatzigeorgiou, A systematic review of studies of web portals for patients with diabetes mellitus. Mhealth 3 (2017), 23.

[20] Fraccaro Paolo, Vigo Markel, and Balatsoukas Panagiotis, et al., Patient Portal Adoption Rates: A Systematic Literature Review and Meta-Analysis. Stud Health Technol Inform 245 (2017), 79-83.

[21] J.Y. Zhao, B. Song, and E. Anand, et al., Barriers, Facilitators, and Solutions to Optimal Patient Portal and Personal Health Record Use: A Systematic Review of the Literature. AMIA Annu Symp Proc 2017 (2017), 1913-1922.

[22] H.-R. Han, K.T. Gleason, and C.-A. Sun, et al., Using Patient Portals to Improve Patient Outcomes: Systematic Review. JMIR Hum Factors 6 (2019), e15038.

[23] C.S. Kruse, K. Bolton, and G. Freriks, The effect of patient portals on quality outcomes and its implications to meaningful use: a systematic review. J Med Internet Res 17 (2015), e44.

[24] L.L. Beal, J.M. Kolman, and S.L. Jones, et al., Quantifying Patient Portal Use: Systematic Review of Utilization Metrics. J Med Internet Res 23 (2021), e23493. 